\title{
Lösungsmöglichkeiten nach fehlgeschlagenen Osteosynthesen: Brust- und Lendenwirbelsäule
}

\author{
Rudolf Beisse, Volker Bühren
}

\section{Zusammenfassung}

Die Therapie von Fehlschlägen nach Eingriffen an der Wirbelsäule stellt für den Wirbelsäulenchirurgen eine Herausforderung dar. Ursächlich sind die häufig komplexen Problemstellungen, die sich aus dem Zusammentreffen mehrerer Komplikationen, wie Infekt, Korrekturverlust oder Fehllage von Implantaten ergeben. Die räumliche Nähe der Rumpfwirbelsäule zu den großen Gefäßen und dem Rückenmark erfordert es, eine mögliche Mit- beteiligung im Rahmen der präoperativen Diagnostik zu erfassen und diese in die Planung des Revisionseingriffs mit einzubeziehen. Das Ziel des Revisionseingriffs ist eine definitive und möglichst weitgehende Wiederherstellung der Stabilität und Form der Wirbelsäule, wobei Funktionseinschränkungen aufgrund der im Vergleich zur primären Stabilisierung meist längerstreckigen Konstruktionen nicht immer vermieden werden können.

\section{Einleitung}

Lange Zeit galten Verletzungen der Brustund Lendenwirbelsäule als Domäne der konservativen Behandlung. Mit der Entwicklung potenter Implantate auch für den traumatologischen Sektor der Wirbelsäulenchirurgie konnte erstmals das in der Behandlung von Extremitätenverletzungen schon lange gültige Prinzip der offenen anatomischen Reposition, der Stabilisierung und frühfunktionellen Behandlung auch in diesem Bereich verwirklicht werden. Auf der Grundlage verbesserter diagnostischer Möglichkeiten und der Einführung praxisorientierter Klassifikationen, wie der AO-Klassifikation nach Magerl [6] wurden allgemein anerkannte Vorgehensweisen der operativen Behandlung entwickelt, die eine weite Verbreitung gefunden haben.

Eine Schattenseite dieser erfreulichen Entwicklung stellen die Fehlschläge nach operativer Behandlung dar, deren Lösung aufgrund der komplexen anatomischen Verhältnisse im Bereich der Wirbelsäule und der umgebenden Strukturen hohe Anforderungen an den behandelnden Chirurgen stellt.

OP-JOURNAL 2004; 20: 92-95

(c) Georg Thieme Verlag KG Stuttgart · New York
Dabei sind Fehlschläge nach Operationen im Wirbelsäulenbereich keine Seltenheit. So beträgt die Versagerquote bei Fusionsoperationen aufgrund degenerativer Veränderungen im lumbalen Bereich 37\%, bei nicht fusionierenden Eingriffen 30\% [4]. Auf traumatologischem Gebiet rechnen wir mit Fusionsraten zwischen 76 und $85 \%$. Etwa die Hälfte der nicht zur knöchernen Ausheilung gekommenen Fälle bedarf eines Revisionseingriffs. Die Angaben aus der Literatur zur Häufigkeit und zum Ausmaß von Schraubenfehllagen nach dorsaler Instrumentierung sind sehr unterschiedlich. Ihre Häufigkeit wird mit Raten zwischen 7 und $40 \%$ angeben. Fehllagen, die um mehr als $6 \mathrm{~mm}$ nach medial von der Ideallage abweichen, sind meist mit neurologischen Ausfällen verbunden und bedürfen in jedem Fall der Revision [3,9].

\section{Hauptteil}

Genauso wie wir die frische Verletzung der Wirbelsäule hinsichtlich der Verletzungsform und der hieraus resultierenden Instabilität analysieren, bedarf auch der Revisionseingriff einer gründlichen Vorbereitung und Diagnostik, die den aktuellen Zustand sowohl klinisch als auch radiologisch wiedergibt. Stabilisierende oder rekonstruktive Eingriffe an der Wir- belsäule führen meist nur im ersten Anlauf zu einem optimalen Ergebnis und jeder weitere korrigierende Eingriff zieht größere Verlusten an Knochensubstanz und Stabilität nach sich, die nur durch langstreckige und aufwändige Konstruktionen wettgemacht werden können.

Eine sorgfältige präoperative Analyse des eingetretenen Schadens und Planung des Revisionseingriffs sind deshalb erforderlich.

\section{Präoperative Diagnostik und Planung (Abb. 1)}

Wird die Indikation zum Revisionseingriff an der Wirbelsäule gestellt, werden sich Operateur und Patient auf eine zeitlich und lokal ausgedehnte Operation einstellen, die erhöhte Anforderungen an die körperliche Verfassung des Patienten stellt. Das Spektrum der Behandlungsmöglichkeiten wird nicht selten durch das Lebensalter oder Begleiterkrankungen limitiert. Da es sich meist um einen Wahleingriff handelt, ist im Einzelfall der zu erwartende Blutverlust abzuschätzen und die Indikation zur Eigenblutspende zu prüfen.

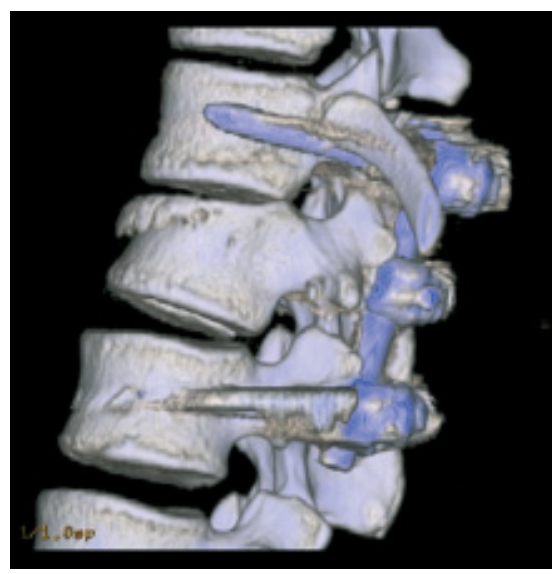

Abb.1 3D-Rekonstruktion der Fehllage einer Pedikelschraube bei Fraktur des LWK 1 Typ A 3.1. 
Im Vordergrund der Abklärung der lokalen Situation an der Wirbelsäule steht die Frage des Vorliegens eines Infektes oder einer bedrohlichen Fehllage von Implantaten in Bezug zu den großen Gefäßen und dem Spinalkanal [7]. Neben konventionellen Röntgenaufnahmen führen wir eine Computertomographie des betroffenen Wirbelsäulenabschnitts und der benachbarten gesunden Anschlusssegmente in Schichten von höchstens $3 \mathrm{~mm}$ durch, um einerseits das Ausmaß von Knochendefekten abzuschätzen und andererseits verankerungsfähige Strukturen für eine geplante Instrumentierung zu finden.

Nicht jede von der Norm abweichende Lage von Schrauben, auch wenn sie die mediale Wand des Spinalkanals überschreiten, stellt per se eine Indikation zum Revisionseingriff dar.

Von einer Fehllage von Implantaten können wir jedoch sprechen, wenn aus ihr eine Bedrängung oder gar Verletzung nervaler Strukturen oder der Gefäße resultiert oder eine fehlende knöcherne Verankerung vorliegt [2].

Die unmittelbare Nähe der großen Gefäße zur Rumpfwirbelsäule nahezu im gesamten Bereich der Rumpfwirbelsäule stellt bei Revisionseingriffen ein großes Gefahrenpotenzial dar (Abb.2). Wird die Rate von Gefäßkomplikationen beim Primäreingriff mit $0,08 \%$ erstaunlich niedrig beziffert [5], so scheint das Risiko bei Revisionseingriffen eher höher zu liegen [1]. So können die mit einer Implantatlockerung oder Infektbildung einhergehenden entzündlichen Veränderungen und Verschwielungen zu einer Verlagerung der Gefäße bis hin zur Arosion führen. Derartige Veränderungen der anatomischen Situation sind auf konventionellen Röntgenaufnahmen nicht und auf

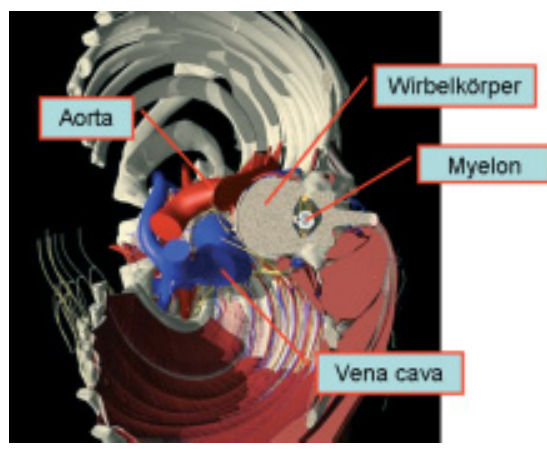

Abb. 2 Topographische Beziehung von Wirbelsäule, Spinalkanal und großen Gefäßen auf Höhe des thorako lumbalen Übergangs. konventionellen ComputertomographieAufnahmen manchmal nur schwer auszumachen. Hier kann eine Angio-Computertomographie oder DSA (Digitale Subtraktions-Angiographie) wertvolle Informationen liefern.

\section{Indikationen zum Revisionseingriff}

Einer Aufstellung und Analyse der BG Unfallklinik Halle zufolge finden sich als häufigste Indikationen zu einem Revisionseingriff [8]:

1. OP-taktische primäre Fehler mit Schraubenfehlpositionierungen und Versorgung über zu lange bzw. über zu kurze Instrumentationsstrecken;

2. Klassifikationsfehler von Wirbelsäulenverletzungen und eine inadäquate Rekonstruktion der ventralen Säule;

3. Schicksalhafte Verläufe mit Infektbzw. Defektpseudarthrosen;

4. Indikationen nach Bandscheibenoperation bzw. degenerativen Instabilitäten.

Nach ventraler Rekonstruktion müssen wir zusätzlich zu den oben aufgeführten implantatbezogenen Komplikationen auch mit den Folgen der höheren $\mathrm{Zu}-$ gangsmorbidität der ventralen Eingriffe rechnen, wozu die Störungen der Innervation der Bauchwand und die Zwerchfellhernie gehören. Mit Ausnahme der Innervationsstörungen handelt es sich durchgängig um operationsbedürftige Komplikationen.

\section{Behandlungsziele}

Das Behandlungsziel richtet sich nach der zugrunde liegenden Komplikation, wobei nicht selten eine Kombination mehrerer Problemstellungen vorliegt. So ist eine Implantatlockerung nicht selten mit einem Infekt assoziiert oder die Schraubenfehllage mit einem Duraleck oder einer Gefäßarosion. Zu den wesentlichen Zielen aller operativer Maßnahmen im Rahmen des Korrektureingriffs gehört die

- Wiederherstellung der Stabilität;

- Ausheilung eines Infektes;

- Wiederherstellung der Form der Wirbelsäule;

- Beseitigung von Einengungen des Wirbelkanals;

- Beseitigung potentieller Gefährdungen benachbarter Strukturen (Gefäße).

Bei der Behandlung von Infektionen an der Wirbelsäule kommt der Wiederherstellung stabiler Verhältnisse absolute Priorität zu. Eine fehlgeschlagene Osteosynthese an der Wirbelsäule konfrontiert den behandelnden Chirurgen nur in den seltensten Fällen mit einer einzigen Komplikation, meist findet er eine komplexe Situation vor, für die keine Standardlösung vorgegeben ist. Umso wichtiger ist es in der Vorbereitung des Eingriff alle anamnestischen und diagnostischen Möglichkeiten nach Art einer Checkliste abzuarbeiten und den Eingriff im Detail präoperativ „durchzuspielen“, um unliebsame Überraschungen intraoperativ weit gehend auszuschließen.

Die folgenden 2 Fallbeispiele sollen dies in der Praxis demonstrieren:

\section{Fall 1}

Problemstellung

Der 46-jährige Patient erlitt bei einem Motorradunfall einen Flexions-Subluxationsbruch A 2.3 mit kneifzangenartiger Fraktur des 12.Brustwirbelkörpers ohne neurologische Ausfälle. Nach zunächst dorsaler Aufrichtung und Stabilisierung mit einem Fixateur interne kam es zur Ausbildung eines Infekts. Nach mehrfacher Revision unter Belassen des Implantats weiterhin Keimnachweis und Infektzeichen. Es folgte der Ausbau des Implantats und die Infektberuhigung.

Nachfolgend kam es zur Ausbildung einer schmerzhaften posttraumatischen Kyphose von $25^{\circ}$ (Abb.3a).

Präoperative Diagnostik und Planung

Allgemeinzustand: guter, altersentsprechender Trainingszustand, keine Einschränkung des kardiopulmonalen Status.

Labor: CRP 1,45 mg/dl, Leuko 6700/nl, Hb $12,7 \mathrm{~g} / \mathrm{dl}$.

Konventionelle Röntgendiagnostik und Computertomographie des thorako lumbalen Übergangs: postraumatischer Kyphosewinkel 25을 Scheitelpunkt BWK 12, Klaffen der Dornfortsatzreihe (Abb. 3b).

Geplant und durchgeführt wurde ein einziger dorso-ventro-dorsaler Korrektureingriff in mehreren Schnitten:

1. Schritt: Dorsale Instrumentierung BWK 11 auf LWK 1.

2. Schritt: Rechtseitenlagerung, thorakoskopischer Zugang zum thorakolumbalen Übergang mit Teilablösung des Zwerchfells und endoskopischem Anterior Release im Bandscheibenraum 


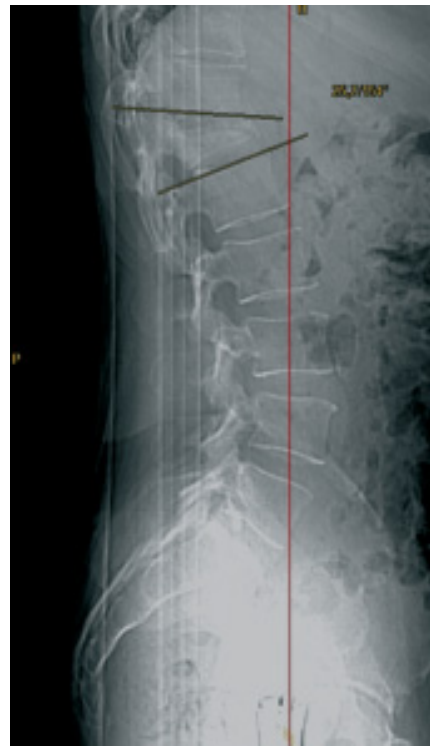

Abb. 3a Korrekturverlust und Posttraumatische Kyphose von 25 nach dorsaler Stabilisierung einer BWK 12-Fraktur Typ B 2.3 mit nachfolgender Infektsituation dorsal und ventral.

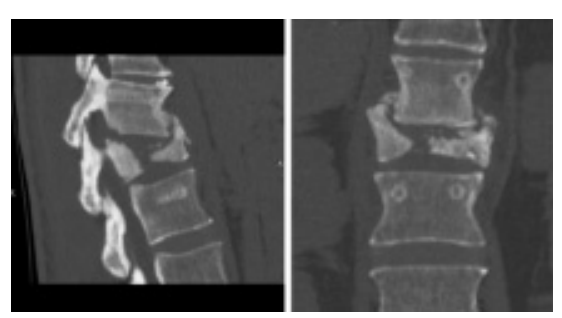

Abb.3b Sagittale und frontale 2D-Rekonstruktion.

BWK 11/12 und BWK 12/ LWK 1 mit Teil-korporektomie BWK 12 [Abb.3c].

3. Schritt: Als Wirbelkörpersatz verwendeten wir einen Synex ${ }^{\circledR}$-Korb, dessen Hohlraum mit einem resorbierbaren Antibiotikaträger (Gentamycin ${ }^{\circledR}$ ) gefüllt und mit Beckenkammspongiosa umlagert wurde. Die Fehlstellung wurde weitgehend durch Distraktion ventral und Kompression dorsal korrigiert. Es erfolgte eine ventrale Instrumentierung und die Fixierung des Fixateur interne dorsal mit. Einlage eines resorbierbaren Antibiotikaträgers (Gentamycin $^{\circledR}$ ) dorsal (Abb.3d u.e).

Die histologische Untersuchung zeigte Knochen- und Knorpelgewebe mit Nekrosen, chronisch-granulierender, teils eitriger Entzündung.

Bakteriologisch fand sich ein hochsensibler Staphycoccus aureus, z. B. auf Gentamycin.

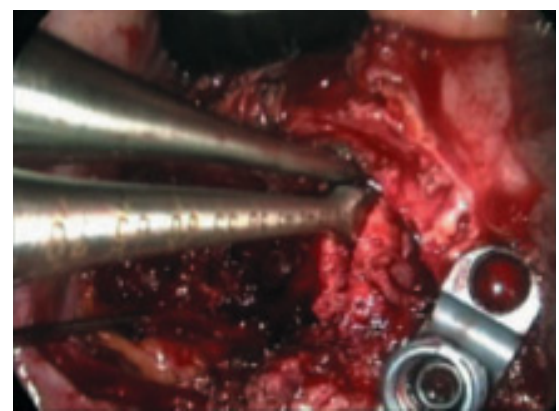

Abb.3c Endoskopisches Anterior Release Bandscheibenfach BWK 11/12.
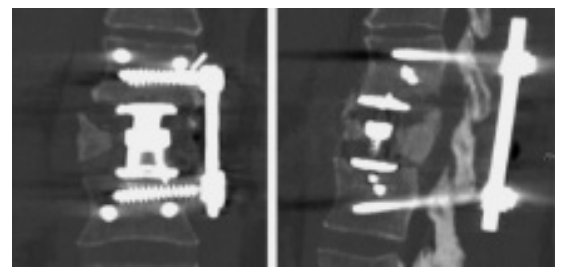

Abb.3d Sagittale und frontale 2 D-Rekonstruktion nach dorso-ventralem Release und Aufrichtung.

Verlauf: Komplikationsloser Heilverlauf, seit 10 Monaten infektfrei.

\section{Fall 2}

Problemstellung

Der 34-jährige Patient verunfallte beim Mountainbiking und zog sich dabei eine Flexions-Distraktionsverletzung Typ B 2.3 des 7. Brustwirbels mit Beteiligung der Hinterkante jedoch ohne Neurologie zu. An der erstversorgenden Klinik erfolgt die dorsale Stabilisierung mit einem Fixateur von BWK 6 auf 8. Das postoperative CT zeigt die Pedikelschrauben zu stark konvergierend, daraufhin erfolgte eine Revision der Schraubenlage zwei Tage später. Dabei kam es bei der Instrumentierung des BWK 6 auf der linken Seite zur starken Blutung aus dem Pedikalkanal als auch zur Blutung in die linksseitige Thoraxhöhle. Bei der linksseitigen Thorakotomie findet sich laut OP-Bericht eine Blutung aus der Aorta, die durch Naht versorgt wird. Die Pedikelschraube BWK 6 links wurde entfernt und der BWK 5 in die dorsale Stabilisierung mit einbezogen. Linksseitig wurde eine Fixation von BWK 5 auf 8, rechts von BWK 6 auf 8 vorgenommen. Postoperativ bestand eine Sensibilitätsminderung am rechten Oberschenkel und Schwäche des rechten Beines.

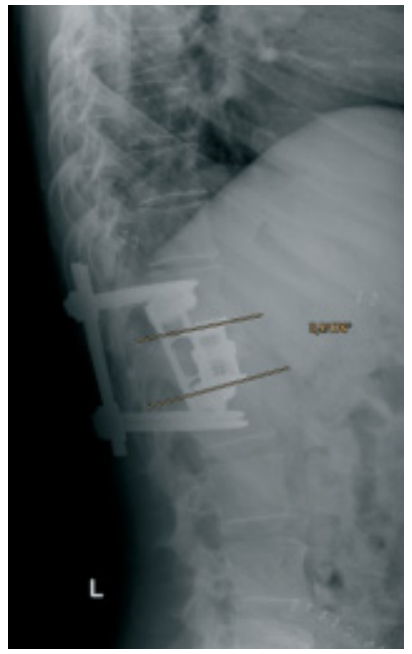

Abb.3e Dorso-ventrale-dorsale Rekonstruktion mit USS ${ }^{\circledR}$-Fixateur interne dorsal und endoskopische Teilkorporektomie und Release ventral, Wirbelkörperersatz und ventraler Instrumentierung (Synex ${ }^{\circledR}$-Korb, MACS TL $\left.{ }^{\circledR}\right)$, Kyphosewinkel $4^{\circ}$.

Bei der Vorstellung in unserer Klinik zeigt sich eine komplexe Fehllage von drei der insgesamt 4 Pedikelschrauben.

Präoperative Diagnostik und Planung

Allgemeinzustand: 34-jähriger Patient, sportlich trainiert, sehr guter Allgemeinzustand.

Labor: CRP $<0,1 \mathrm{mg} / \mathrm{dl}$, Leuko 7300/nl, $\mathrm{Hb} 17,3 \mathrm{~g} / \mathrm{dl}$, insgesamt keine Infektzeichen.

Befund der konventionellen Röntgendiagnostik und Computertomographie der Brustwirbelsäule (Abb.4a):

1. BWK 5: Pedikelschraube liegt links neben dem Pedikel, rechter Pedikel unbesetzt.

2. BWK 6: Pedikelschraube links entfernt, Gefäßclips aortennah, rechte Pedikelschraube verläuft durch den Spinalkanal mit Bedrängung des Myelons.

3. BWK 7: keilförmig deformiert mit zentralem Knochendefekt.

4. BWK 8: linksseitige Pedikelschraube liegt mit ihrer Spitze der Aortenwand an - bzw. ein $\rightarrow$ weitere Abklärung durch CT-Angio erforderlich.

Ergebnis der CT-Angio: Die im linken Pedikel BWK 8 liegende Schraube hebt mit ihrer Spitze die Aorta von der Wirbelkörperwand $a b$, die Spitze perforiert möglicherweise die Aortenwand (Abb.4b). 


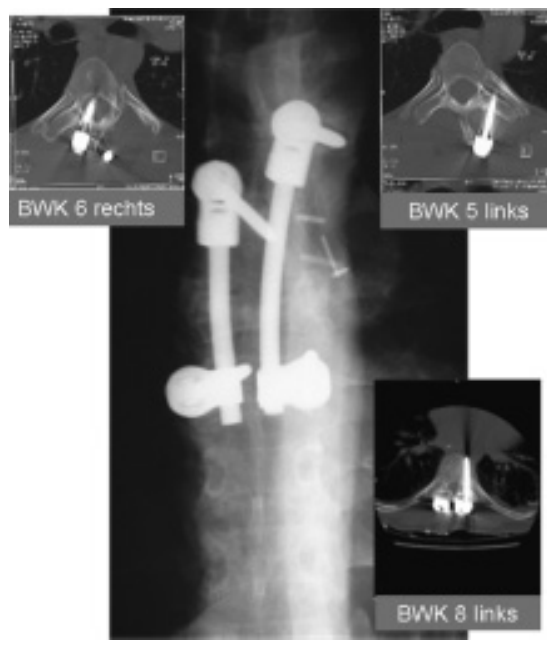

Abb.4a Dorsale Stabilisierung einer BWK 7Fraktur Typ B2.3 mit intraspinaler Lage einer Pedikelschraube und Irritation der Aortenwand durch eine Pedikelschraube.

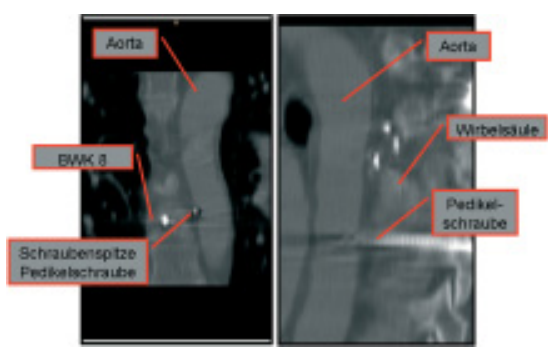

Abb.4b CT-Angiographie mit Darstellung der gedeckten Läsion der Aortenwand in beiden Ebenen.

Planung und Durchführung eines zweizeitigen dorsoventralen Korrektureingriffs in Kooperation mit der Thoraxund Gefäßchirurgie des Klinikums Großhadern, München:

1. Schritt: Einbringen eines AortenStents über die linke Leiste durch den Gefäßchirurgen (Abb.4c).

2. Schritt: Entfernung der in die Aortenwand einliegenden Schraube im BWK 9 unter dem Schutz des Stents und Entfernung der im Spinalkanal liegenden Schraube unter Sicht nach Hemilaminektomie, um ein „Aufwickeln“ der Dura und des Myelons beim Herausdrehen zu vermeiden.

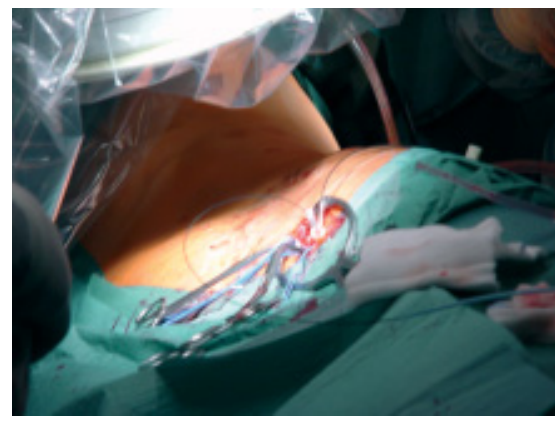

Abb.4c Einbringen eines Aorten-Stents über die linke Leiste vor Entfernung der aortennahen Schraube.

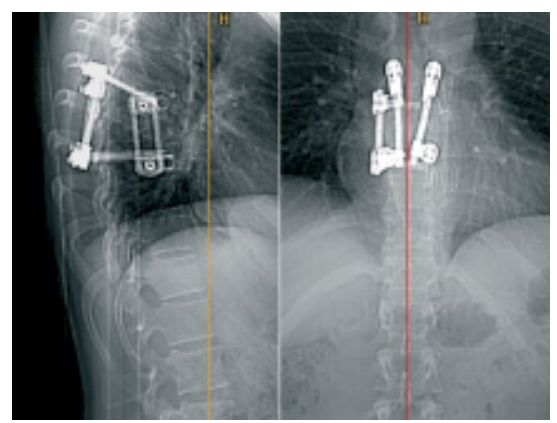

Abb.4d Postoperatives Ergebnis nach Entfernung der intraspinal gelegenen Schraube über Hemilaminektomie sowie der aortennahen Schraube unter Schutz des Stents, Neuanlage des Fixateur interne (USS ${ }^{\circledR}$ ) mit anschließender thorakoskopischer ventraler Rekonstruktion (MACS TL ${ }^{\circledR}$ ) und Spanfusion BWK 6 auf 8.

3. Dorsale Re-Stabilisierung mit Universal Spine System ${ }^{\circledast}$.

4. Schritt: Ventraler thorakoskopischer Eingriff von rechts mit Teilkorporektomie BWK 8, Interposition eines Beckenkammspans und ventraler Instrumentierung mit einem MACS TL ${ }^{\circledR}$-System (Abb. 4d).

Postoperativ kam es zum komplikationslosen Heilverlauf mit vollständiger Rückbildung der neurologischen Ausfälle und Symptome.

\section{Schlussfolgerung}

Das klinisch-radiologische Bild nach Fehlschlägen nach Osteosynthesen an der Wirbelsäule reicht vom posttraumatischen Korrekturverlust über die Implantatlockerung- und Fehllage bis hin zu vital bedrohlichen Zuständen durch mögliche Gefäßkomplikationen. Zur Einschätzung des Ausgangsbefundes und zur Planung des Revisionseingriff bedarf es einer subtilen diagnostischen Vorarbeit. Der Eingriff selbst führt idealerweise zur infektfreien Wiederherstellung von Form und Stabilität der Wirbelsäule, wobei aufgrund meist längerstreckiger Versorgungen eine bleibende Bewegungseinschränkung nicht immer vermeidbar ist.

\section{Literatur}

1 Beisse R, Potulski M, Bühren V. Endoscopic Techniques for the Management of Spinal Trauma. Eur J Trauma 2001; 27: 275 - 291

2 Blauth M, Knop C, Bastian L. Brust-und Lendenwirbelsäule: Springer-Verlag, 1997

3 Castro WHM, Halm H, Jerosch J et al. Accuracy of pedicle screw placement in lumbar vertebrae. Spine 1996; 21: 1320-1324

4 Eichholz KM, Ryken TC. Complications of Revision Spinal Surgery. Neurosurg Focus 15, 2003

5 Faciszewski T, Winter RB, Lonstein JE et al. The surgical and medical perioperative complications of anterior spinal fusion. Surgery in the Thoracic and lumbar spine in adults. Spine 1995; 20: 1592-1599

6 Magerl F, Aebi S, Gertzbein SD et al. A comprehensive classification of thoracic and lumbar injuries. Eur Spine J 1994; 3: 184 201

7 Margulies JY, Aebi M, Farcy JP. Revision Spine Surgery. St. Louis: Mosby 1999

8 Roehl K, Weidt F, Becker SWJ. Indikationen für Revisionsoperationen der Wirbelsäule. Z Orthop Ihre Grenzgeb 2001

9 Sjöström L, Jacobsson O, Karlstrom G et al. CT analysis of pedicles and screw tracts after implant removal in thoracolumbar fractures. J Spinal Disord 1993; 6: 225-231

Ao. Prof./Utah Dr. med. Rudolf Beisse Leitender Arzt

Prof. Dr. med. Volker Bühren

Ärztlicher Direktor

Abteilung Chirurgie/Unfallchirurgie BG-Unfallklinik Murnau

Prof.-Küntscher-Str. 8

D-82418 Murnau 